\title{
HCI AND ITS EFFECTIVE USE IN DESIGN AND DEVELOPMENT OF GOOD USER INTERFACE
}

\author{
Ishrat begum \\ Assistant professor, Government First Grade college, Raichur, India
}

\begin{abstract}
Systems that are designed better are used better. Even the fastest and most powerful systems are of no use until people understand them and use with ease. HCI plays a vital role in understanding the need of users and designing the user interfaces in accordance with the user requirements. Since there is an alarming increase in internet and World Wide Web users, it is important that usable and useful systems are built so that even the new users without prior knowledge of computers too can use it with ease and are able to benefit from the information that is now available. This paper is an attempt to bring forward the need of good user interface designs and implementation with HCI principles that offer solutions to build better and improved interfaces that are more natural to use and simple to adapt than the existing interfaces.
\end{abstract}

Keywords: HCI-Human Computer Interaction, User Interface, user-centered design, star life cycle model, Ubiquitous computing ****

\section{INTRODUCTION}

Human-computer interaction [HCI] is a discipline concerned with the study, design, construction and implementation of human-centric interactive computer systems [1]. A user interface such as a GUI is how a human interacts with computer, and HCI goes beyond designing screens and menus that are easier to use and studies the reasoning behind building specific functionality into computers and the long term effects that systems will have on humans [1]. HCI covers a very broad spectrum of philosophies, perspectives and many types of expertise regarding computer development .whereas computer science is concerned with application design and engineering of the human interfaces; sociology and anthropology are concerned with the interactions between technology, work and organization and the way that human systems and technical systems mutually adapt to each other[2].

The User interface (UI) can be defined as a medium through which the user and the computer interact with each other. UI interactions are different with different systems. For ex. digital watches have buttons that are pressed to set time, Microwaves ovens have dials to turn on or a digital display or touchpad etc. to set the timer for cooking. PCs have a screen, a keyboard, a mouse or joystick or trackball to interact with the system. Hence different systems have different user interfaces that may be easy to learn and adapt or difficult to use for the users [9].

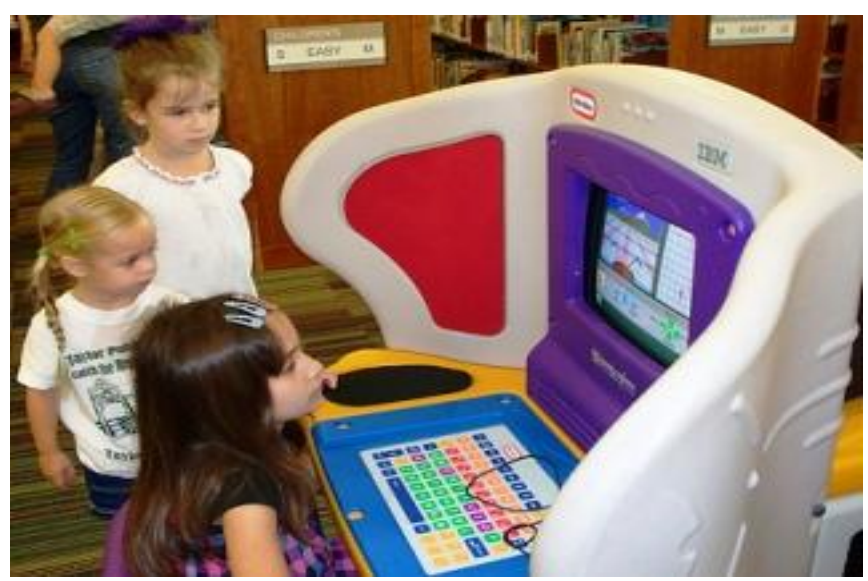

Fig-1: A user interface designed specifically for children. (Image courtesy of taylor library on Flickr.)[16]

\section{RESEARCH ON USER INTERFACE (UI)}

\subsection{Advancement of User Interface (UI)}

The evolution of user interface design ideas began in the early 1980s with a messy desk metaphor, popularized by Apple Macintosh, where files and folders were spread around the display screen and it was a perfect incubator for the developing paradigm of graphical interfaces. As HCI developed, there was a move from the desktop in three distinctive senses. First, the desktop metaphor proved to be challenging with increased icons of files and folders. Later by the mid-1990s, HCI experts and others understood that search was a more fundamental feature than browsing for finding 
things in a user interface. During the same time with early World Wide Web pages appearance the cluttered desktop metaphor was removed and an advance step in usability was seen (like telnet and ftp). The second sense in which HCI moved ahead of desktop was through the attractiveness of internet on computing and on society. Email emerged as one of the most important HCI applications, which made people to interact with each other through computers. Equipments and applications like instantaneous messaging, Wikis, blogs, online forums, media services, and other combined systems were available.[3] Thus social computing started growing rapidly. Third way in which HCI moved ahead was through the volatile diversification in the natural science of devices. Before desktop applications were consolidated, new kinds of devices like laptops, handheld devices appeared. One leading edge today is the ubiquitous or everywhere computing: the constant absorption of computing into human surroundings like cars, home appliances ,furniture, clothing etc .Desktop computing is no matter what still very important with its own benefits. The goal of HCI has moved beyond the desktop and its focus will continue to move. The contributions of HCI are never ending as it will investigate, develop and exploit new areas of possibilities not merely as technologies or designs, but also as a means of enhancing human activity and experience.

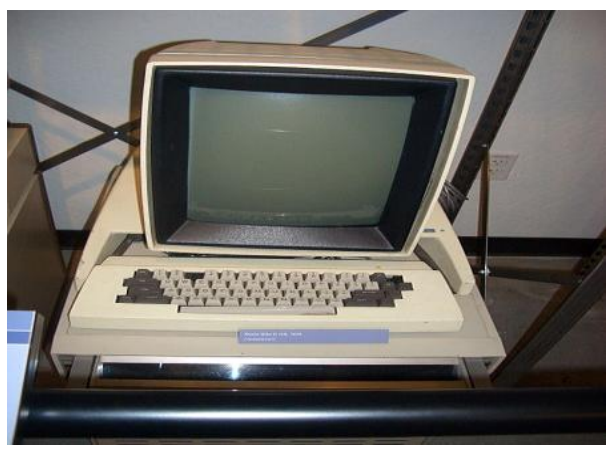

Fig-2: Xerox Alto had an early GUI[3].

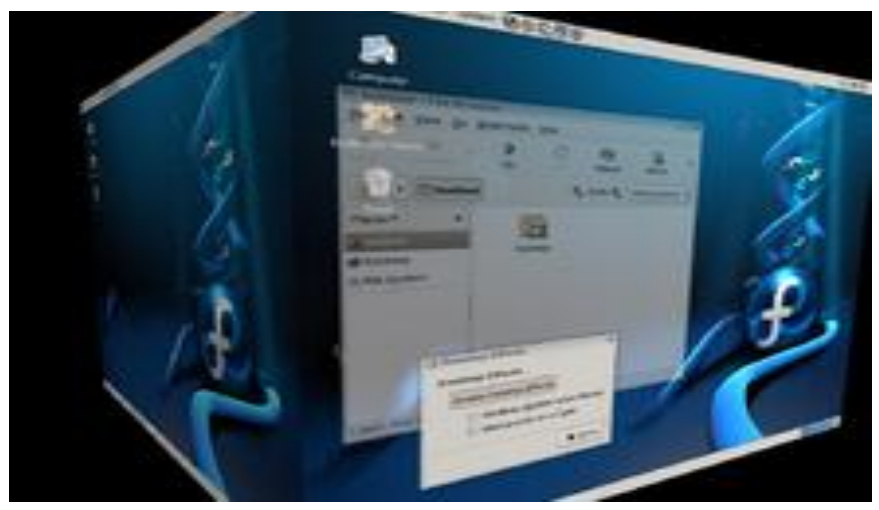

Fig-3: compiz running on Fedora Core 6 with AIGLX (1984) today's 3D User Interface the Reactable: a multitouch [3].

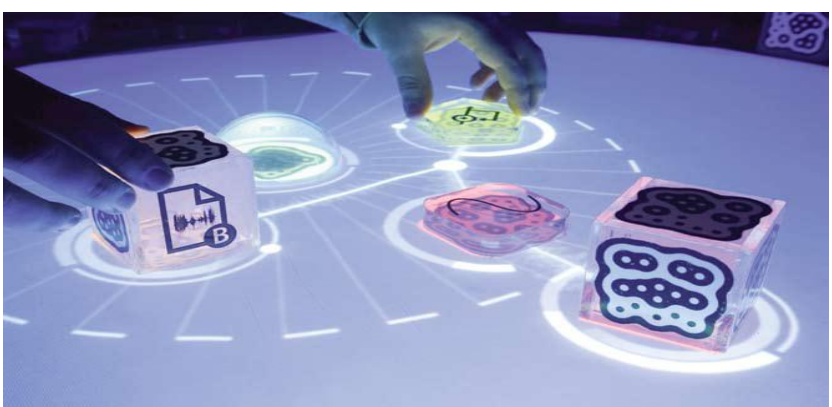

Fig-4: The Reactable: a multitouch interface for playing music. Performers can simultaneously interact with it by moving and rotating physical objects on its surface. [9]

In the past few years new input techniques are developed that are far better and have removed the shortcomings of mouse and keyboard interaction. For ex. Tablet computers use stylusbased interaction on a screen, and paper based systems that digitally capture markings on special paper using a camera embedded in a pen [9]. Also developments from multi-touch surfaces facilitating interaction with hands and fingers on touch sensitive screens, allow one to manipulate objects as if they are physical. From GUIs to multi-touch, speech to gesturing has evolved the ways we interact with computers easily today. The change to multiple points of input supports original forms of interaction where people can share a single interface by gathering around it and interacting together (fig.4)[9].Similar growth is also seen with touchable interfaces, where everyday physical objects are embedded with computation, sensing and reacting to the ways they are selected up, manipulated and moved in space.Eye movements have been used for the past many years as means to support the disabled in interacting with computers. Recently the initiation of 'braincomputer interfaces' which allow people with severe physical disabilities to use their brain waves to interact with their environments the real time brainwave activity is beginning to be used to control digital movies, turn on music, switch the lights on and off. These interfaces can even control robot arms, allowing paralyzed individuals to manipulate objects [9].

\subsection{Need for Good Interface Design [4]}

$>$ Nearly $40-60 \%$ of today's software is involved in user interface management with high interactivity with the users-end user programming.

$>$ User interactivity is getting more complex with-3D graphics and virtual reality and increased reality activities.

$>$ Bad interface leads to many problems with introducing and maintaining a system.

$>$ Users get frustrated and reject systems they don't understand.

$>$ Users are comfortable and less frustrated with systems that are easy to use and easy to understand. 
$>$ Users are prone to make more errors with systems that are difficult to use.

$>$ A good user interface reduces coding costs, lessen high costs of interface problems, reduce serious life threatening errors, and sell more products and leads to popularity of computers and more

\subsection{Reasons for Poor Interface Design [4]}

$>$ Lack of HCI based training of people developing interfaces.

$>$ A range of knowledge is required to design good interfaces

$>$ Rapid technological advances is required to design

$>$ Companies reluctant to commit resources.

$>$ Poor management-where programmers are not thinking and designing as users.

$>$ Lack of interaction and involvement of users.

\subsection{Usability}

The two terms that are important to HCI are functionality and usability. Functionality of a system can be defined as a set of actions or services that are provided to its users. Functionality has its importance only when it becomes possible to be used efficiently by the user [15].The effectiveness of a system is achieved only when there is a proper balance between the functionality and usability [14]. The ISO 9241-11 (1998) defines usability as the "extent to which a product can be used by specified users to achieve specified goals with effectiveness, efficiency and satisfaction in a specified context of use" [13]. The Usability objectives are as follows:

- Utility - product enables user to attain their goals the tasks that it was designed to carry out and/or wants needs of user.

- Efficiency (ease of use) - quantitatively calculated by speed of performance or error rate and is coupled to a percentage of users.

- Learn ability - user's ability to operate the system to some defined level of competence after some predestined period of training. It also refers to the ease with which new users can begin effective interaction and achieve maximum performance.

- Attitude (approach) - user's perceptions, feelings and opinions about the product, are usually captured through both written and oral communication.

- Robustness-the level of support given to the user to achieve and assess the goal directed behavior [7].

- Predictability-determinism and operation visibility. User actions should be matched by a response and affordance/logical constraints should be used to indicate available actions [7].

- Synthesizability-It can assess effect of past actions

$>$ Direct manipulation interfaces promise immediate honesty, others have eventual honesty
Command line interfaces are never honest.

- Generalizability-extending specific interaction knowledge to new situations

$\checkmark$ Helps give a predictive model of system for user

$\checkmark$ A form of uniformity ex. drawing circles! drawing ellipses

$\checkmark$ Applications should provide cut/paste/copy operations wherever required [7].

\section{DESIGNING FOR USERS}

A good user interface caters to the needs of end users and supports them in the tasks they wish to undertake.Attributes of a good user interface is its easy to adapt, to learn with minimal training requirements, has few errors, helps in error recovery, flexible and makes the system useful. Computer systems should be designed for the needs and capabilities of the users for whom they are intended [7]. The goal of UCD is to produce products that have a high degree of usability.

\subsection{User-centered Design [8]}

An approach that involves users throughout the design and development process in user interface is 'User centered design' process (UCD). This is also called as Human centered design process. It is general term for a philosophy and variety of methods like usability Testing, usability engineering, heuristic evaluation, discount evaluation, and participatory design which focus on designing computer systems with users involvement[17]. There is a spectrum of ways in which users are involved in UCD in one or another way. This approach focuses on understanding users of a computer system under development and tasks that the users will perform. The UCD principles as stated in Handbook of Usability Testing: How to Plan, Design, and Conduct Effective Tests, John Wiley and Sons, Inc., 1984 by Jeffery Rubin [6] are:

1. Early focus on users and tasks

a. Structured and systematic information gathering (consistent across the board)

b. Designers trained by experts before conducting data collection sessions

2. Empirical Measurement and testing of product usage

a. Focus on ease of learning and ease of use

b. Testing of prototypes with actual users

3. Iterative Design

a. Product designed, modified and tested repeatedly.

b. Allow for the complete overhaul and rethinking of design by early testing of conceptual models and design ideas.

The UCD approach ensures that the users are involved as a part of HCI design and development process. It enhances the usability of the final product. When compared with classic life 
cycle or water fall model each phase is an independent part of software development which must be completely satisfied before switching to the next phase. Where as the user centered design approach is based on users participation throughout the design life cycle and it is also highly iterative so that the design can be tested (or evaluated) to meet the user requirement. Where as the basic life cycle model performs evaluation at the end of the process [10]

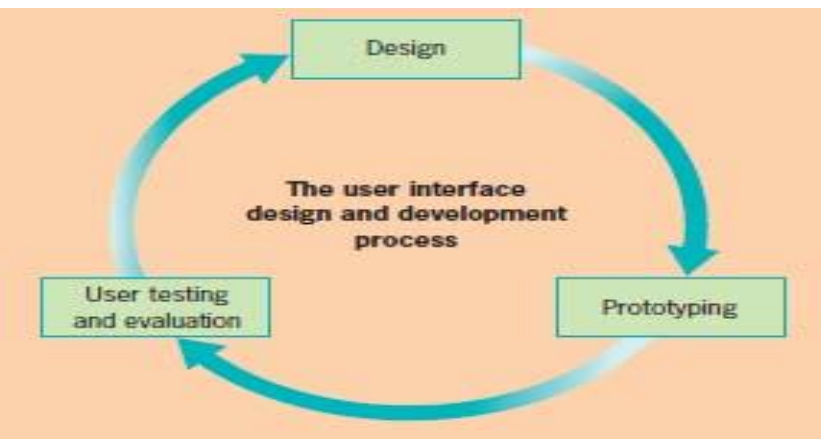

Fig-5: The iterative user interface design and evaluation process. (From Greenberg, 1996)[11]

\subsection{Star Life Cycle Making the Design Process}

\section{Iterative}

An approach adapted from a model proposed by Hix an Hartson (1993), known as the star life cycle model as shown In fig-6 encourages iteration [5]. The central point of the star model is evaluation which is important at all stages in the life cycle unlike the classic life cycle which evaluates at the end. The role of evaluation is to always check the design and improve at all stages. Evaluation techniques like interviews with users and others, observation of user work place, use of questionnaires and other types of surveys are included. The star life cycle is "intended to support equally both top-down and bottom-up development and inside-out, outside-in development"(Hix an Hartson, 1993, P.101). Thus, in the star life cycle design can start with any process.

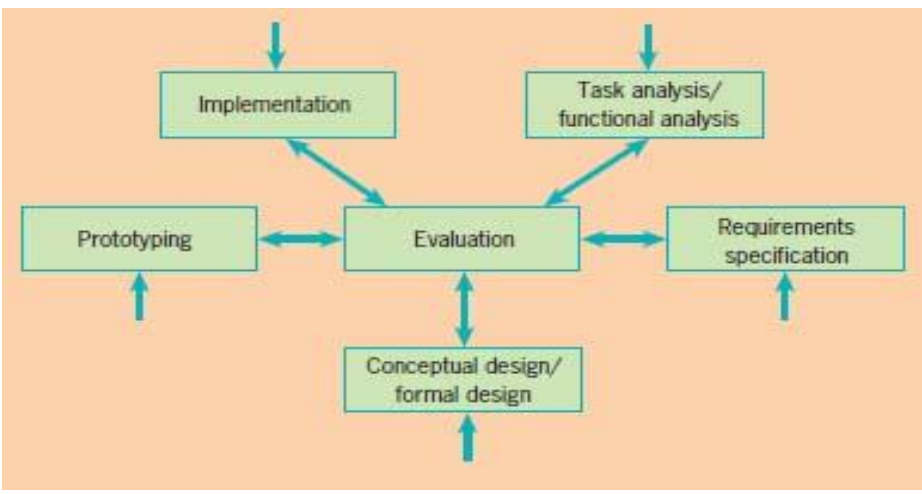

Fig-6: The star life cycle model. (From Hix and Hartson, 1993) [5] [11].

\subsection{HCI Extends the Research and Design Cycle [9]}

As we know that the UCD model follows a typical iterative cycle comprising four fundamental processes in which we study, design, build and evaluate technology [9].HCI has taken an initiative and proposed a new agenda to extend this design by adding one more stage, which entails conceptual analysis and this stage is labeled as 'understand'. For understanding a problem has been a part of the study phase. This initial and new stage focuses on human values and pinpoints those that we wish to design for and to research. This stage ultimately helps to understand the user's domain, environments and cultures. It provides a framework to guide design and research. These investigations will be helpful to carry further research in stage 2.Thus in stage 2 a grounded understanding of human values of interest are brought out through interaction, taking into account social factors, environmental factors and so on. Thus sketching out different technological possibilities. Further stage 3, Design stage is primarily a design or creative phase and involves the design goals. Stage 4 , is the Build phase involves techniques from low-methods such as paper prototypes and sketches, to more hi-tech, robust systems ready for long term field testing. There are various tools and technologies available for researchers to understand many kinds of building tasks quickly and easily. The final stage is of evaluation of what has been built .Here various HCI methodologies can be used.Thus this HCI's conventional design model considers the human values that the researchers are trying to implement or research [9].

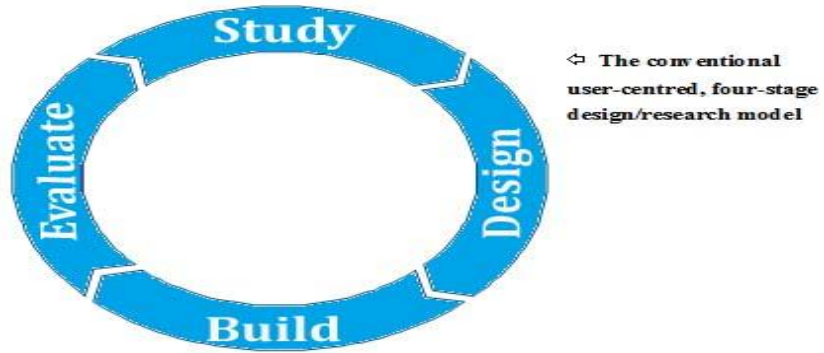

Fig-7: The conventional user-centered, four-stage design model [9].

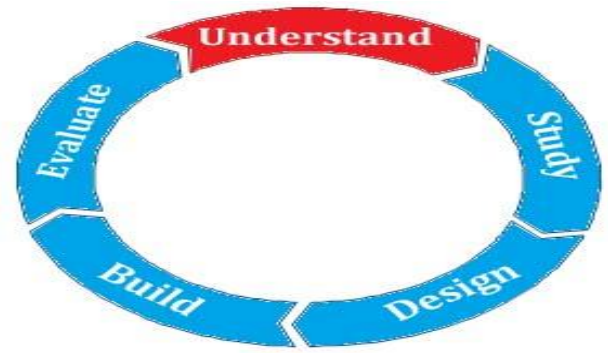

Fig-8: Extended user-centered, five stage design/research model. The new stage entails conceptual analysis or 'understanding' [9]. 


\section{CONCLUSIONS}

Computer systems should be designed in such a way that they full fill the needs and capabilities of the users and UCD is such an approach that optimizes a computer systems usability.UCD design helps to assess how usable the product will be as usability is the prime determiner for a software success. While designing interfaces we find how important it is to follow an iterative user-centered design process. The role of evaluation is always to improve the design at all stages of the life cycle. In this paper we discuss the importance of a good interface design and the impact of bad UI Design. The importance of user involvement is essential to develop a good UI. We can conclude the two types of knowledge should be taken into account when designing user interface i.e, information and user interface design knowledge (Design principles and design rules). HCI has emerged as a unique research area, the methods and techniques have changed the UI design from desktop to GUI and gestures[9]. Since every day technology is moving at a faster rate there is an increasing demand for fast and user friendly interfaces which can not only understand and fulfill the requirements of different users but also give them satisfaction with effectiveness. New ways of understanding the users are being explored with an extension of user centered design approach. The new methods must complement the existing methods of designing and stress on usability between prototypes and engineered solutions. In future more advanced light weight, rapid prototyping and design iteration processes will be required leading to simplified existence of relation between Human and machine. HCI professional need to go beyond the scientific community they live in and explore the society as a total [9].Thus this paper has attempted to survey and give an overview of importance and need of good interface design with the support of HCI principles.

\section{REFERENCES}

[1] http://www.webopedia.com/TERM/H/HCI.html

[2] D. Te'eni, J. Carey and P. Zhang, Human Computer Interaction: Developing Effective Organizational Information Systems, John Wiley \& Sons, Hoboken (2007

[3] http://en.wikipedia.org/wiki/History_of_the_graphical_ user_interface

[4] Information Systems Analysis and Design $\square 2001$ Marilyn Mantei and John Mylopoulos,Interface Design $--54$

[5] H.R. Hartson and D. Hix, Developing User Interfaces, John Wiley, New York, 1993.

[6] J. Rubin, Handbook of Usability Testing, John Wiley \& Sons, 1994.

[7] David Aspinall,Interface Design Rules, lecture HCI lecture-2 Informatics, University of Edinburgh,23rd October 2007

[8] Introducing user-Interface Design"Web accessibility initiative",http://www.w3.org/WAI/redesign/ucd.
[9] Being Human: Human-Computer Interaction in the year 2020,Editors: Richard Harper, Tom Rodden, Yvonne Rogers and Abigail Sellen ISBN: 978-09554761-1-2

[10] Rogers, Y, Sharp, H, and Preece, J (2007) Interaction Design : Beyond Human Computer Interaction. 2nd ed. Hoboken, NewJersey: Wiley.

[11] Introducing user interface design, http://booksite.elsevier.com/9780120884360/casestudie s/Chapter_01.pdf

[12] D.J. Mayhew, Principles and Guidelines in Software User Interface Design, Prentice Hall,Englewood Cliffs, 1992.

[13] Debbie Stone, Caroline Jarrett, Mark Woodroffe, Shailey Minocha, User Interface Design and Evaluation, Morgan Kaufmann, 29-Apr-2005.

[14] J. Nielsen, Usability Engineering, Morgan Kaufman, San Francisco (1994).

[15] B. Shneiderman and C. Plaisant, Designing the User Interface: Strategies for Effective Human-Computer Interaction (4th edition), Pearson/Addison-Wesley, Boston (2004).

[16] www.Google.co.in

[17] Abras,Krichmar,Preece,"User-centered design”.Available at: http://www.phoenix.goucher.edu/ jillz/cs325.../Usercentered-design-Article.doc

[18] D.A. Norman, "Design Principles for HumanComputer Interfaces," Proc. Computer-Human Interaction Conf. (CHI 83), ACM Press, 1983.

[19] B.A. Myers, "A brief history of human-computer interaction technology", ACM interactions, 5(2), pp 4454 (1998). 